Journal of Algebra Combinatorics Discrete Structures and Applications

\title{
Note on the permutation group associated to E-polynomials*
}

\section{Hirotaka Imamura, Masashi Kosuda, Manabu Oura}

\begin{abstract}
This is a continuation of our project which focuses on E-polynomials and the related combinatorics. A pair of groups appearing in the definition of E-polynomials yields the permutation group. In this paper, we determine the multi-matrix structures of the centralizer algebras of the tensor representations of this permutation group.
\end{abstract}

2010 MSC: 05E10, 05E30

Keywords: Centralizer algebra, Permutation group

\section{Introduction}

The purpose of this note is to investigate the centralizer algebras of the tensor representation of the finite group $G$ that arises from E-polynomials in genus 1. We also discuss the association scheme which appears as the first case. Our results can be regarded as combinatorial properties of E-polynomials.

The study of Eisenstein series is of interest because of its importance in number theory. We defined and studied finite analogue of Eisenstein series from combinatorial point of view. We call it an Epolynomial in some of our previous papers $[8,10,11]$

Let $g$ be a positive integer. Then, an action of $\Gamma_{g}=S p(2 g, \mathbf{Z})$ on the theta series induces a finite subgroup $H_{g}$ of $G L\left(2^{g}, \mathbf{C}\right)$. It is known that the invariant ring of $H_{g}$ is closely related to the ring of modular forms for $\Gamma_{g}$. As to this direction in higher genus, see [9, 14]. There exist some results based on the fact that the weight enumerator of a self-dual and doubly even binary code is invariant under the action of $H_{g}[3,4,13,15]$. The correspondence existing among the invariant theory of the finite groups,

\footnotetext{
* This work was supported by JSPS KAKENHI Grant Numbers 17K05164, 19 K03398.

Hirotaka Imamura; PFU Limited, Japan (email: h.i.ishikawa01@gmail.com).

Masashi Kosuda; Department of Engineering, Yamanashi University, Japan (email: mkosuda@yamanashi.ac.jp). Manabu Oura (Corresponding Author); Institute of Science and Engineering, Kanazawa University, Japan (email: oura@se.kanazawa-u.ac.jp).
} 
the theory of modular forms, and combinatorics such as coding theory motivates the notion and study of E-polynomials.

Let $K_{g}$ be a subgroup of $H_{g}$ fixing $x_{0}$. An E-polynomial of degree $\ell$ in degree $g$ is defined by

$$
\varphi_{\ell}\left(x_{a}: a \in \mathbf{F}_{2}^{g}\right)=\frac{1}{\left|H_{g}\right|} \sum_{K_{g} \backslash H_{g} \ni \sigma}\left(\sigma x_{0}\right)^{\ell} .
$$

The rings generated by E-polynomials have been studied in [10, 11] (cf. [8]). Moreover, the representation theory of $H_{1}$ is investigated and the centralizer rings of the tensor representation of $H_{1}$ are determined in [6]. Since the character table of $H_{1}$ is used in this study, it is reproduced at the end of this paper from [6].

As usual, let $\mathbf{C}$ denote the complex number field. We denote by $\mathcal{M}_{d}$ the matrix algebra of degree $d$ over C. For simpliticy, let $n \mathcal{M}_{d}$ denote $\underbrace{\mathcal{M}_{d} \oplus \cdots \oplus \mathcal{M}_{d}}_{n}$.

\section{Preliminaries}

We follow the notations in [6].

Let $H_{1}$ be the group of order 96 generated by $\frac{1+i}{2}\left(\begin{array}{cc}1 & -1 \\ 1 & 1\end{array}\right),\left(\begin{array}{ll}1 & 0 \\ 0 & i\end{array}\right)$ and $K_{1}=\left\langle\left(\begin{array}{ll}1 & 0 \\ 0 & i\end{array}\right)\right\rangle$ be its subgroup of orer 4 . A set of representatives of $K_{1} \backslash H_{1}$ is given by

$$
\begin{gathered}
1, T, T^{2}, T^{3}, T^{4}, T^{5}, T^{6}, T^{7}, T D, T D^{2}, T D^{3}, T^{3} D, T^{3} D^{2}, T^{3} D^{3}, \\
T^{5} D, T^{5} D^{2}, T^{5} D^{3}, T^{7} D, T^{7} D^{2}, T^{7} D^{3}, T D^{2} T, T^{3} D^{2} T, T^{5} D^{2} T, T^{7} D^{2} T
\end{gathered}
$$

and we name each of the classes $1,2, \ldots, 24$, for example, $24=K_{1} T^{7} D^{2} T$. Now, we set $\Omega=\{1,2, \ldots, 24\}$, then the action of $H_{1}$ on $\Omega$ gives a transitive permutation representation of $H_{1}$. We observe that $T$ and $D$ correspond to

$$
t=(1,2,3,4,5,6,7,8)(9,14,12,17,15,20,18,11)(10,21,13,22,16,23,19,24),
$$

and

$$
d=(2,9,10,11)(4,12,13,14)(6,15,16,17)(8,18,19,20)(21,22,23,24)
$$

respectively. Consequently, we obtain a faithful permutation representation $G=\langle t, d\rangle$ of $H_{1}$. The group $G$ is an imprimitive permutation group of order 96 on the 24 points. Under the correspondence

$$
g \mapsto\left(\delta_{\alpha^{g}, \beta}\right)_{\alpha, \beta \in \Omega},
$$

we may regard $G$ as a matrix group.

\section{Results}

We follow the argument presented in the papers $[6,7]$. Let us denote by $\chi$ the permutation character of $G$. It is known that $\chi(g)$ is the number of $\alpha \in \Omega$ which is fixed by $g$.

Proposition 3.1. $\chi=\chi_{1}+\chi_{5}+\chi_{9}+\chi_{10}+\chi_{12}+\chi_{13}+\chi_{14}+\chi_{15}+\chi_{16}$. 
Proof. Let

$$
\chi=m_{1} \chi_{1}+m_{2} \chi_{2}+\cdots+m_{16} \chi_{16} .
$$

Here we remind that $\chi_{1}$ is the identity character of $G$. Since the characters depend on the conjugacy classes, $\chi(\mathfrak{C})$ denotes the value of $\chi$ at a conjugacy class $\mathfrak{C}$. Then, we have that

$$
\left(\begin{array}{llll}
\chi\left(\mathfrak{C}_{1}\right) & \chi\left(\mathfrak{C}_{2}\right) & \ldots & \chi\left(\mathfrak{C}_{16}\right)
\end{array}\right)=\left(\begin{array}{llll}
m_{1} & m_{2} & \ldots & m_{16}
\end{array}\right) \mathbf{X},
$$

where $\mathbf{X}$ denotes the character table of $G$ presented at the end of this paper. If we know the explicit values $\chi\left(\mathfrak{C}_{\mathfrak{i}}\right)$ 's on the left-hand side, we multiply $\mathbf{X}^{-1}$ on both sides from the right to get the result.

Suppose $g \in \mathfrak{C}$ for a conjugacy class $\mathfrak{C}$, then $\chi(\mathfrak{C})$ is the number of $\alpha \in \Omega$ which is fixed by $g$. Therefore the values of $\chi\left(\mathfrak{C}_{i}\right)$ 's can be given by

$$
\left(\chi\left(\mathfrak{C}_{1}\right) \quad \chi\left(\mathfrak{C}_{2}\right) \ldots \chi \chi\left(\mathfrak{C}_{16}\right)\right)=(24,0,0,0,0,0,4,0,0,0,0,0,4,0,4,0) .
$$

This completes the proof of Proposition 3.1.

Before proceeding further, we shall interpret this proposition from the perspective of Wielandt [17]. Let $\mathfrak{A}$ be the centralizer ring of the matrix group $G$. Then Proposition 3.1 shows that $\mathfrak{A}$ is commutative and of dimension 9 and a basis can be obtained as follows. Let $G_{1}$ be the stabilizer of a point $1 \in \Omega$ in $G$. Then $G_{1}$ has 9 orbits

$$
\{1\},\{3\},\{5\},\{7\},\{2,9,10,11\},\{4,12,13,14\},\{6,15,16,17\},\{8,18,19,20\},\{21,22,23,24\} .
$$

With each orbit $\Delta$ we associate a matrix $\mathfrak{V}(\Delta)=\left(v_{\alpha, \beta}^{\Delta}\right)$ by

$$
v_{\alpha, \beta}^{\Delta}= \begin{cases}1 & { }^{\exists} g \in G \text { such that } 1^{g}=\beta \text { and } \alpha^{g^{-1}} \in \Delta, \\ 0 & \text { otherwise. }\end{cases}
$$

In particular, for $\Delta=\{1\}$, we have that

$$
\mathfrak{V}(\{1\})=I
$$

where $I$ is the $24 \times 24$ identity matrix. Then the matrices $\mathfrak{V}(\Delta)$ 's form a basis for $\mathfrak{A}$.

Next we shall connect $\mathfrak{A}$ with the theory of association schemes ([1]. cf. [16]). Under the ordering of the orbits given above, we denote the matrices $\mathfrak{V}(\Delta)$ by $A_{0}=I, A_{1}, \ldots, A_{8}$. We observe that the matrices $A_{0}, A_{2}, A_{8}$ are symmetric and

$$
{ }^{t} A_{1}=A_{3},{ }^{t} A_{4}=A_{7},{ }^{t} A_{5}=A_{6} .
$$

Then the set of matrices $\mathcal{X}=\left\{A_{0}, A_{1}, \ldots, A_{8}\right\}$ forms a non-symmetric commutative association scheme of class 8 . The algebra $\mathfrak{A}$ is also called the Bose-Mesner algebra of the association scheme $\mathcal{X}$. With regards to the commutativity and the normality of $A_{i}$ 's, $\mathcal{X}$ has another basis $E_{0}=\frac{1}{24} J, E_{1}, \ldots, E_{8}$ of the primitive idempotents, where $J$ is a $24 \times 24$ matrix with every entry 1 . The transformation matrix $P$ is defined by

$$
\left(\begin{array}{llll}
A_{0} & A_{1} & \ldots & A_{8}
\end{array}\right)=\left(\begin{array}{llll}
E_{0} & E_{1} & \ldots & E_{8}
\end{array}\right) P
$$

which can also be called the first eigenmatrix of $\mathcal{X}$. In our case, the first eigenmatrix is

$$
\left(\begin{array}{ccccccccc}
1 & 1 & 1 & 1 & 4 & 4 & 4 & 4 & 4 \\
1 & 1 & 1 & 1 & -2 & -2 & -2 & -2 & 4 \\
1 & 1 & 1 & 1 & 0 & 0 & 0 & 0 & -4 \\
1 & i & -1 & -i & 2+2 i & -2+2 i & -2-2 i & 2-2 i & 0 \\
1 & i & -1 & -i & -1-i & 1-i & 1+i & -1+i & 0 \\
1 & -i & -1 & i & 2-2 i & -2-2 i & -2+2 i & 2+2 i & 0 \\
1 & -i & -1 & i & -1+i & 1+i & 1-i & -1-i & 0 \\
1 & -1 & 1 & -1 & 2 i & -2 i & 2 i & -2 i & 0 \\
1 & -1 & 1 & -1 & -2 i & 2 i & -2 i & 2 i & 0
\end{array}\right) .
$$


It turns out that $\mathcal{X}$ is isomorphic to No.349 in the list [5].

Summing up, we have obtained the following theorem.

Theorem 3.2. The centralizer ring of the transitive permutation group $G_{1}$ on the 24 points is a commutative association scheme of degree 8 which is isomorphic to No.349 in [5].

We proceed to the centralizer algebra of the tensor representation of $G$. Let $\mathfrak{A}^{(k)}$ be the centralizer algebra of the $k$-th tensor representation of $G$. Notice $\mathfrak{A}=\mathfrak{A}^{(1)}$. We set

$$
\chi^{\otimes k}=\sum_{i=1}^{16} d_{i}^{(k)} \chi_{i}
$$

and

$$
\overrightarrow{d^{(k)}}=\left(d_{1}^{(k)}, d_{2}^{(k)}, \ldots, d_{16}^{(k)}\right)
$$

We are going to determine the coefficients $d_{i}^{(k)}$ explicitly. By Proposition 3.1, we already knew

$$
\overrightarrow{d^{(1)}}=(1,0,0,0,1,0,0,0,1,1,0,1,1,1,1,1)
$$

We shall consider the matrix $A$ such that

$$
\left(\begin{array}{c}
\chi \chi_{1} \\
\chi \chi_{2} \\
\vdots \\
\chi \chi_{16}
\end{array}\right)=A\left(\begin{array}{c}
\chi_{1} \\
\chi_{2} \\
\vdots \\
\chi_{16}
\end{array}\right)
$$

The matrix $A$ can be calculated explicitly as

$$
\begin{aligned}
A & =\text { X.diagonal }\left(\left[\chi\left(\mathfrak{C}_{1}\right), \chi\left(\mathfrak{C}_{2}\right), \ldots, \chi\left(\mathfrak{C}_{16}\right)\right]\right) . \mathbf{X}^{-1} \\
& =\text { X.diagonal }([24,0,0,0,0,0,4,0,0,0,0,0,4,0,4,0]) . \mathbf{X}^{-1} \\
& =\left(\begin{array}{llllllllllllllll}
1 & 0 & 0 & 0 & 1 & 0 & 0 & 0 & 1 & 1 & 0 & 1 & 1 & 1 & 1 & 1 \\
0 & 1 & 0 & 0 & 1 & 0 & 1 & 1 & 0 & 0 & 1 & 0 & 1 & 1 & 1 & 1 \\
0 & 0 & 1 & 0 & 0 & 1 & 1 & 0 & 0 & 1 & 1 & 1 & 1 & 0 & 1 & 1 \\
0 & 0 & 0 & 1 & 0 & 1 & 0 & 1 & 1 & 0 & 1 & 1 & 0 & 1 & 1 & 1 \\
1 & 1 & 0 & 0 & 2 & 0 & 1 & 1 & 1 & 1 & 1 & 1 & 2 & 2 & 2 & 2 \\
0 & 0 & 1 & 1 & 0 & 2 & 1 & 1 & 1 & 1 & 2 & 2 & 1 & 1 & 2 & 2 \\
0 & 1 & 1 & 0 & 1 & 1 & 2 & 1 & 0 & 1 & 2 & 1 & 2 & 1 & 2 & 2 \\
0 & 1 & 0 & 1 & 1 & 1 & 1 & 2 & 1 & 0 & 2 & 1 & 1 & 2 & 2 & 2 \\
1 & 0 & 0 & 1 & 1 & 1 & 0 & 1 & 2 & 1 & 1 & 2 & 1 & 2 & 2 & 2 \\
1 & 0 & 1 & 0 & 1 & 1 & 1 & 0 & 1 & 2 & 1 & 2 & 2 & 1 & 2 & 2 \\
0 & 1 & 1 & 1 & 1 & 2 & 2 & 2 & 1 & 1 & 3 & 2 & 2 & 2 & 3 & 3 \\
1 & 0 & 1 & 1 & 1 & 2 & 1 & 1 & 2 & 2 & 2 & 3 & 2 & 2 & 3 & 3 \\
1 & 1 & 1 & 0 & 2 & 1 & 2 & 1 & 1 & 2 & 2 & 2 & 3 & 2 & 3 & 3 \\
1 & 1 & 0 & 1 & 2 & 1 & 1 & 2 & 2 & 1 & 2 & 2 & 2 & 3 & 3 & 3 \\
1 & 1 & 1 & 1 & 2 & 2 & 2 & 2 & 2 & 2 & 3 & 3 & 3 & 3 & 4 & 4 \\
1 & 1 & 1 & 1 & 2 & 2 & 2 & 2 & 2 & 2 & 3 & 3 & 3 & 3 & 4 & 4
\end{array}\right)
\end{aligned}
$$

We have thus gotten

$$
\overrightarrow{d^{(k)}}=\overrightarrow{d^{(k-1)}} A \quad(k \geq 2) .
$$

Then we have only to apply linear algebra. The matrix $A$ is diagonalizable and we get ${ }^{1}$

$$
P^{-1} A P=\operatorname{diagonal}([4,4,4,24,0,0,0,0,0,0,0,0,0,0,0,0])
$$

1 The matrices $\mathbf{X}, A$, and $P$ can be found at [12]. 
for some non-singular matrix $P$. We apply this calculation to

$$
\begin{aligned}
\overrightarrow{d^{(k)}} & =\overrightarrow{d^{(1)}} P A^{k-1} P^{-1} \\
& =\left(a_{k}, b_{k}, b_{k}, b_{k}, c_{k}, d_{k}, d_{k}, d_{k}, e_{k}, e_{k}, f_{k}, g_{k}, g_{k}, g_{k}, h_{k}, h_{k}\right),
\end{aligned}
$$

where

$$
\begin{aligned}
& a_{k}=6 \cdot 24^{k-2}+3 \cdot 4^{k-2}, \\
& b_{k}=6 \cdot 24^{k-2}-4^{k-2}, \\
& c_{k}=12 \cdot 24^{k-2}+2 \cdot 4^{k-2}, \\
& d_{k}=12 \cdot 24^{k-2}-2 \cdot 4^{k-2}, \\
& e_{k}=12 \cdot 24^{k-2}+2 \cdot 4^{k-2}, \\
& f_{k}=18 \cdot 24^{k-2}-3 \cdot 4^{k-2}, \\
& g_{k}=18 \cdot 24^{k-2}+4^{k-2}, \\
& h_{k}=24^{k-1} .
\end{aligned}
$$

We have thus obtained the following multi-matrix structures of $\mathfrak{A}^{(k)}$.

Theorem 3.3. We have that

$$
\mathfrak{A}^{(k)} \cong \begin{cases}9 \mathcal{M}_{1} & k=1, \\ \mathcal{M}_{a_{k}} \oplus 3 \mathcal{M}_{b_{k}} \oplus \mathcal{M}_{c_{k}} \oplus 3 \mathcal{M}_{d_{k}} \oplus 2 \mathcal{M}_{e_{k}} \oplus \mathcal{M}_{f_{k}} \oplus 3 \mathcal{M}_{g_{k}} \oplus 2 \mathcal{M}_{h_{k}} & k \geq 2,\end{cases}
$$

where $a_{k}, b_{k}, \ldots, h_{k}$ are given above.

Corollary 3.4. (1) $\mathfrak{A}^{(k)}$ is commutative if and only if $k=1$.

$(2) \operatorname{dim} \mathfrak{A}^{(k)}=3456 \cdot 24^{2(k-2)}+48 \cdot 4^{2(k-2)}(k \geq 1)$.

The second assertion of Corollary 3.4 is obtained by taking the sum of the dimensions of the simple components. It would be interesting if we interpret this number from combinatorial point of view, see [6]. This paper is concluded with a small table of $\operatorname{dim} \mathfrak{A}^{(k)}$.

$$
\begin{array}{c|cccccc}
k & 1 & 2 & 3 & 4 & 5 & 6 \\
\hline \operatorname{dim} \mathfrak{A}^{(k)} & 9 & 3504 & 1991424 & 1146630144 & 660452081664 & 380420288937984
\end{array}
$$

Acknowledgment: This work was supported by JSPS KAKENHI Grant Numbers 17K05164, 19K03398. The computations were done with Magma [2] and Maple.

\section{References}

[1] E. Bannai, T. Ito, Algebraic combinatorics I: association schemes, The Benjamin/Cummings Publishing Co, California (1984).

[2] W. Bosma, J. Cannon, C. Playoust, The Magma algebra system I: the user language, J. Symbolic Comput. 24(3-4) (1997) 235-265.

[3] M. Broué, M. Enguehard, Polynômes des poids de certains codes et fonctions thêta de certains réseaux, Ann. Sci. École Norm. Sup. 5(1) (1972) 157-181.

[4] W. Duke, On codes and Siegel modular forms, Internat. Math. Res. Notices 5 (1993) 125-136. 
[5] A. Hanaki, http://math.shinshu-u.ac.jp/ hanaki/as/.

[6] M. Kosuda, M. Oura, On the centralizer algebras of the primitive unitary reflection group of order 96, Tokyo J. Math. 39(2) (2016) 469-482.

[7] M. Kosuda, M. Oura, Centralizer algebras of the group associated to $\mathbf{Z}_{4}$-codes, Discrete Math. 340(10) (2017) 2437-2446.

[8] T. Motomura, M. Oura, E-polynomials associated to $\mathbf{Z}_{4}$-codes, Hokkaido Math. J. 47(2) (2018) $339-350$

[9] M. Oura, The dimension formula for the ring of code polynomials in genus 4, Osaka J. Math. 34(1) (1997) 53-72.

[10] M. Oura, Eisenstein polynomials associated to binary codes, Int. J. Number Theory 5(4) (2009) $635-640$.

[11] M. Oura, Eisenstein polynomials associated to binary codes (II), Kochi J. Math. 11 (2016) 35-41.

[12] M. Oura, http://sphere.w3.kanazawa-u.ac.jp

[13] B. Runge, On Siegel modular forms I, J. Reine Angew. Math. 436 (1993) 57-86.

[14] B. Runge, On Siegel modular forms II, Nagoya Math. J. 138 (1995) 179-197.

[15] B. Runge, Codes and Siegel modular forms, Discrete Math. 148(1-3) (1996) 175-204.

[16] C. C. Sims, Graphs and finite permutation groups, Math. Z. 95 (1967) 76-86.

[17] H. Wielandt, Finite permutation groups, Academic Press, New York-London (1964). 


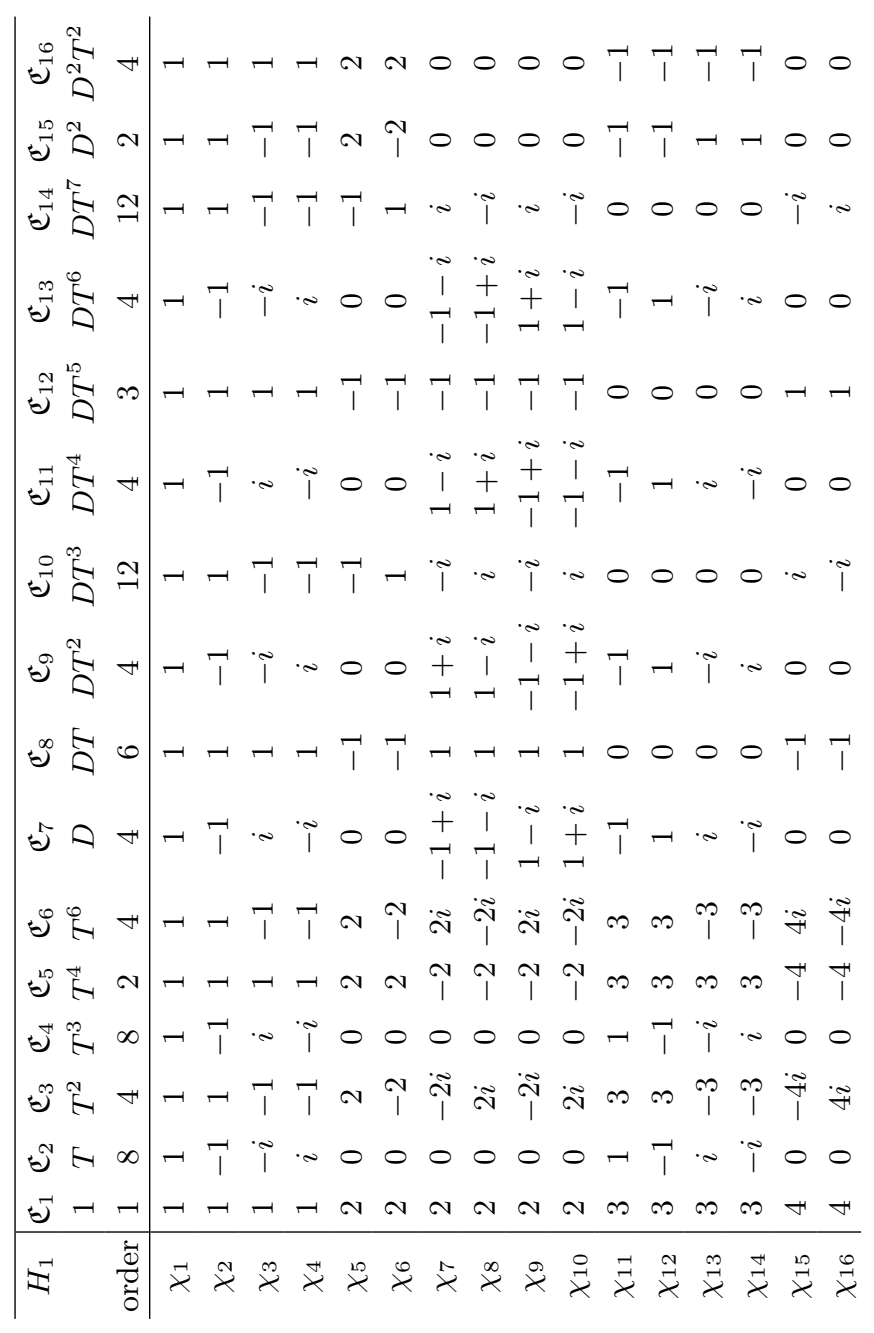

